\title{
Editorial
}

\section{Standards of Reporting: The CONSORT, QUORUM, and STROBE Guidelines}

\author{
Richard A. Brand MD
}

Published online: 19 March 2009

(c) The Association of Bone and Joint Surgeons 2009

Standards of scientific reporting have evolved from the very beginning of scientific reporting. Virtually all journals now publish instructions for authors and most medical journals adhere to certain standards of publication. Such standards have been promoted by international groups such as the International Committee of Medical Journal Editors (ICMJE) and Committee on Publication Ethics (COPE). The ICMJE has published general standards for crafting scientific articles: the "Uniform Requirements for Manuscripts Submitted to Biomedical Journals: Writing and Editing for Biomedical Publication" [7]. These guidelines include suggestions not only for manuscript preparation, but also guidelines for ethical issues related to publishing. COPE, however, focuses on ethical issues [1]. CORR adheres to these guidelines; authors may find links to these guidelines in our online Instructions for Authors.

Such guidelines, as important as they are, lack sufficient detail to ensure all important information is included. Therefore, in addition to these general guidelines for preparing a manuscript, numerous international groups have published guidelines to ensure various sorts of studies contain all essential information. These include the CONSORT (Consolidated Standards of Reporting Trials) guidelines for randomized trials [2, 3, 8], QUORUM (Quality of Reporting of Meta-analyses) [6] and MOOSE (Meta-analysis Of Observational Studies in Epidemiology) [13] for meta-analyses, and STROBE (Strengthening the Reporting of Observational Studies in Epidemiology) for various sorts of observational studies (the majority of clinical studies in surgical disciplines) $[12,15]$. In essence,

R. A. Brand ( $\square)$

Clinical Orthopaedics \& Related Research, 1600 Spruce Street, Philadelphia, PA 19103, USA

e-mail: dick.brand@clinorthop.org these guidelines tell investigators and authors what information is required to ensure readers (and reviewers) can properly evaluate the study.

Despite these standards, most reports of clinical studies lack such critical information. I suspect this is less by intent than lack of awareness of what information is required. In addition, although many journals, including CORR require a Level of Evidence [5, 10, 16] for studies involving patients, most clinical articles published in surgical journals have a relatively low level; prospective, randomized trials are uncommon (and often impractical), and even sufficiently large retrospective cohort studies to control for confounding variables may be unachievable for many conditions or treatments in single institutions. In the absence of high levels of evidence, systematic reviews [9, 11, 17] and meta-analyses [4] have become increasingly common. My search of PubMed for articles limited to metaanalysis yielded 276 articles from 1950-1989, 2116 from 1990-1994, 3736 from 1995-1999, 7920 from 2000-2004, and 9313 from 2005 to 2009. Most of these analyses undoubtedly collected information from lower level studies, thereby incurring the limitations of the individual studies. Virtually all systematic and meta-analyses we have recently published noted missing and variably reported data.

In contrast to medical disciplines, surgical disciplines do not have the advantage of being able to conduct tightly designed prospective, randomized, blinded, controlled trials. (A PubMed search limited to randomized controlled trials suggested The Journal of Bone and Joint Surgery and CORR had together published 556 RCTs and although I did not individually check these for quality, they reflect a small fraction of the articles both journals publish.) For the foreseeable future, most of our information will arise from observational studies rather than prospective trials. In this setting, it is especially important that each article contain all 
relevant information for future systematic reviews and metaanalyses. The STROBE guidelines [12] provide authors with lists of critical information for reporting three sorts of observational studies: cohort (longitudinal studies typically reporting outcomes of treatment in one or more cohorts), case-control (studies identifying factors in outcomes), and cross-sectional studies (studies to identify prevalence of factors or characteristics in a population at a single time).

CORR now requires authors to adhere to CONSORT guidelines for randomized clinical trials, QUORUM guidelines for meta-analyses, and STROBE guidelines for observational clinical studies. Authors will be able to download from our Instructions to Authors convenient templates for the various sorts of articles we publish; these templates contain the information required. In addition, we request authors read and follow the guidelines in the Cochrane Handbook for Systematic Reviews of Interventions 4.2.6 [14]. Adherence to these standards will enhance our ability to answer key clinical questions in more definitive ways than we have in the past, and to answer more questions.

\section{References}

1. 2008 Committee on Publication Ethics: COPE. Annual report. Available at: http://publicationethics.org/annualreport/2008. Accessed January 25, 2009.

2. Altman DG. Better reporting of randomised controlled trials: the CONSORT statement. BMJ. 1996;313:570-571.

3. Begg C, Cho M, Eastwood S, Horton R, Moher D, Olkin I, Pitkin R, Rennie D, Schulz KF, Simel D, Stroup DF. Improving the quality of reporting of randomized controlled trials: the CONSORT statement. JAMA. 1996;276:637-639.

4. Bhandari M, Morrow F, Kulkarni AV, Tornetta P 3rd. Metaanalyses in orthopaedic surgery: a systematic review of their methodologies. J Bone Joint Surg Am. 2001;83:15-24.
5. CEBM Centre for Evidence Based Medicine. Oxford Centre for Evidence-based Medicine Levels of Evidence (May 2001). Available at: http://www.cebm.net/levels_of_evidence.asp. Accessed February 10, 2009.

6. Clarke M. The QUORUM statement. Lancet. 2000;355:756-757.

7. International Committee of Medical Journal Editors. Uniform Requirements for Manuscripts Submitted to Biomedical Journals: Writing and Editing for Biomedical Publication. Available at: http://www.icmje.org/. Accessed January 25, 2009.

8. Moher D, Schulz KF, Altman D; CONSORT Group (Consolidated Standards of Reporting Trials). The CONSORT statement: revised recommendations for improving the quality of reports of parallel-group randomized trials. JAMA. 2001;285:1987-1991.

9. Montori VM, Swiontkowski MF, Cook DJ. Methodologic issues in systematic reviews and meta-analyses. Clin Orthop Relat Res. 2003;413:43-54.

10. Sackett DL. Evidence-based medicine. Semin Perinatol. 1997;21: 3-5.

11. Sterne JA, Egger M, Smith GD. Systematic reviews in health care: investigating and dealing with publication and other biases in meta-analysis. BMJ. 2001;323:101-105.

12. STROBE Statement: STrengthening the Reporting of OBservational studies in Epidemiology. March 30, 2008. Available at: http://www.strobe-statement.org/News\%20Archive.html. Accessed January 25, 2009.

13. Stroup DF, Berlin JA, Morton SC, Olkin I, Williamson GD, Rennie D, Moher D, Becker BJ, Sipe TA, Thacker SB. Metaanalysis of observational studies in epidemiology: a proposal for reporting. Meta-analysis Of Observational Studies in Epidemiology (MOOSE) group. JAMA. 2000;283:2008-2012.

14. The Cochrane Collaboration. Cochrane Handbook for Systematic Reviews of Interventions 4.2.6. Available at: http://www. cochrane.org/resources/handbook/. Accessed January 30, 2009.

15. von Elm E, Altman DG, Egger M, Pocock SJ, Gotzsche PC, Vandenbroucke JP; STROBE Initiative. The strengthening the reporting of observational studies in epidemiology (STROBE) statement: guidelines for reporting observational studies. Lancet. 2007;370:1453-1457.

16. Wright JG, Swiontkowski MF, Heckman JD. Introducing levels of evidence to the journal. J Bone Joint Surg Am. 2003;85:1-3.

17. Wright RW, Brand RA, Dunn W, Spindler KP. How to write a systematic review. Clin Orthop Relat Res. 2007;455:23-29. 